\title{
Are AD-typical regions the convergence point of multiple pathologies?
}

\author{
Sylvia Villeneuve*, Miranka Wirth and Renaud La Joie \\ Helen Wills Neuroscience Institute, University of California, Berkeley, CA, USA \\ *Correspondence: villeneuve.sylvia@gmail.com \\ Edited by: \\ Isidro Ferrer, University of Barcelona, Spain \\ Reviewed by: \\ Willem Huijbers, Harvard Medical School, USA \\ Elizabeth Mormino, Massachusetts General Hospital, USA \\ Michel Grothe, German Center for Neurodegenerative Diseases (DZNE), Germany
}

Keywords: aging, amyloid, tau, vascular, neurodegeneration, Alzheimer's disease

The amyloid hypothesis proposes a serial model of causality whereby beta-amyloid $(\mathrm{A} \beta)$ initiates a cascade of negative events such as neurofibrillary tangle formation leading to neurodegeneration, and eventually clinical onset of Alzheimer's disease (AD). While this hypothesis was mainly founded on genetic forms of AD observations, increasing results coming from $\mathrm{A} \beta$ imaging suggests that the reality for lateonset $\mathrm{AD}$ is more complex. Clearly, the disease develops in an older brain, where age-associated comorbid factors are more prevalent and therefore have a more significant influence on disease expression. Furthermore, it is well established that around one-third of cognitively normal older adults have abnormal $A \beta$ accumulation in their brain (Aizenstein et al., 2008), indicating that $A \beta$ alone might not be sufficient to lead to the clinical expression of late-onset $\mathrm{AD}$. Most of the late-onset AD cases might therefore be the consequence of multifactorial pathologies (Chételat, 2013).

Alzheimer's disease is associated with a characteristic pattern of macroscopic neurodegeneration (that can be detected in vivo using MRI and FDG PET biomarkers) in limbic and heteromodal regions of the cerebral cortex, here referred to as AD-typical regions (Dickerson et al., 2009; Landau et al., 2009; Schroeter and Neumann, 2011; La Joie et al., 2012; Wirth et al., 2013a). In this opinion paper, we argue that multiple factors work together with $\mathrm{A} \beta$ to hasten neurodegeneration in these limbic and heteromodal brain regions. Specifically, we propose that brain regions typically found to be atrophied and/or hypometabolic in $\mathrm{AD}$ dementia are vulnerable to multiple, and at least partly independent, pathologies (e.g., $A \beta$, tau, and vascular factors) and therefore represent regions where the impact of these pathologies converges (Figure 1). We further suggest that some of these pathologies might interact (i.e., have a synergistic effect) in $\mathrm{AD}$-typical regions and that most of $A \beta$-related neurodegeneration might in fact be the consequence of these interactions. This hypothesis would explain why some individuals show cognitive impairment with relatively low levels of $A \beta$, while others have very high levels of $A \beta$ without cognitive deficits. We therefore suggest that even if $A \beta$ might be a needed pathological feature of lateonset $\mathrm{AD}$ clinical expression, its harmful effect might depend on other pathological factors that could emerge independently. This viewpoint thus emphasizes the idea that multiple pathways can trigger $\mathrm{AD}$ typical atrophy/ hypometabolism and contribute to the clinical expression of AD. While these pathways can be due to AD or non-AD factors, the convergence of these "other" pathways with $\beta$-amyloidosis might be needed for the development of cognitive deficits (Wirth et al., 2013a; Mormino et al., 2014) and clinical progression to dementia (Knopman et al., 2012).

It became evident in the past years that $\mathrm{A} \beta$ is not the only factor driving neurodegeneration in $\mathrm{AD}$-typical regions (Fjell et al., 2013; Wirth et al., 2013b). This suggests that other factors may work with $\mathrm{A} \beta$ to cause the brain changes typically found in patients with AD. Neurofibrillary tangles, which consist of microtubule-associated protein tau, are the other hallmark of
AD. Even though the amyloid cascade hypothesis postulates that $A \beta$ leads to tau pathology, neurofibrillary pathology can develop independently and prior to $\mathrm{A} \beta$ accumulation (Spillantini and Goedert, 2013). "Primary age-related tauopathy" (PART) has recently been proposed to describe a pathology that is commonly observed in the brains of older individuals (Crary et al., 2014). From this perspective, $A \beta$ and tau can increase the risk of $\mathrm{AD}$ via independent mechanisms that work together to induce synaptic and neuronal loss (Small and Duff, 2008). This idea does not exclude the possibility that $\mathrm{A} \beta$ can induce tau pathology, rather it suggests that tau pathology can occur independently of $\mathrm{A} \beta$ and that individuals who have PART might be more vulnerable to $A \beta$ if the latter starts to accumulate.

Recent work has proposed that $\mathrm{A} \beta$ and tau, measured by CSF levels, interact to trigger neurodegeneration in $\mathrm{AD}$-typical regions such as the temporoparietal associative cortex (Fortea et al., 2014) and the entorhinal cortex (Desikan et al., 2014). Furthermore, medial frontal thinning associated with CSF p-tau seems to be present only in subjects with abnormal levels of $\mathrm{A} \beta$ (Fortea et al., 2014). Based on these interactions and because tau pathologies preferentially affect the temporal lobe (Braak and Braak, 1991; Whitwell et al., 2008), we suggest that temporoparietal $\mathrm{AD}$-typical regions represent points of convergence between $A \beta$ and tau pathologies (Figures 1B,C). Even if neocortical association areas are not primarily affected by tau accumulation (or its impact), they represent key regions where $A \beta$ 


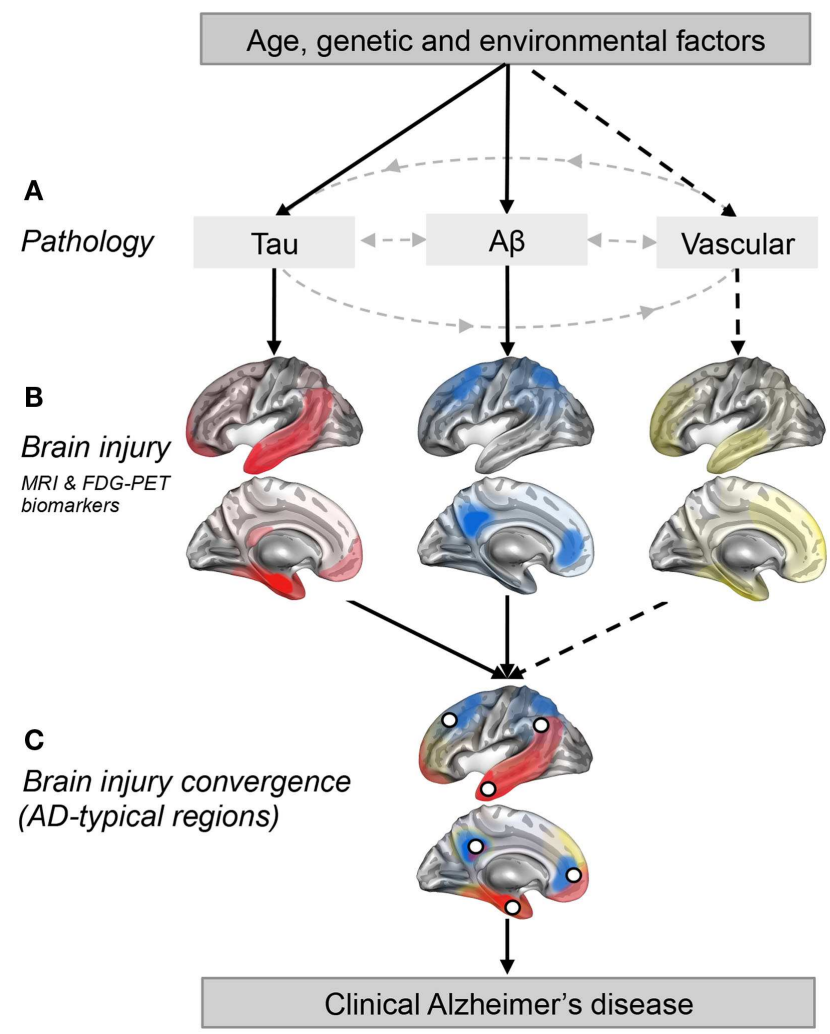

FIGURE 1 | Regional convergence of different pathologies (and their impact) involved in the clinical expression of late-onset Alzheimer's disease. (A) Solid lines represent pathologies that are needed to develop the clinical onset of Alzheimer's disease (AD), while the dotted line represent a pathological factor that is not needed but, if present increases the risk of developing AD. (B) The pattern of brain injury associated with tau, $A \beta$, and vascular pathologies is represented by a schematic illustration based on current literature (Braak and Braak, 1991; Whitwell et al., 2008; Debette et al., 2011; La Joie et al., 2012; Villeneuve et al., 2014). (C) White dots represent brain regions where at least two pathologies are converging. These white dots also represent brain regions that are typically found to be atrophied and/or hypometabolic in individuals with AD (Dickerson et al., 2009; Landau et al., 2009; Schroeter and Neumann, 2011; Wirth et al., 2013a), and when atrophied in cognitively normal older adults, they increase the risk of progression to $A D$ (Dickerson et al., 2009).

accumulates, and are probably one of the first regions were both pathologies meet (since the transentorhinal cortex is mainly spared from $A \beta$ ). This point of convergence of both pathologies might be what triggers their synergetic impact on brain integrity.

Cerebrovascular disease (e.g., cerebral microbleeds, white matter lesions, infarcts) and vascular risk factors (e.g., hypertension, dyslipidemia, and diabetes) are prevalent in older individuals and are known to increase the risk of $\mathrm{AD}$ (Prins and Scheltens, 2015). Even if such factors are not needed for the development of $\mathrm{AD}$, they seem to increase the risk of $\mathrm{AD}$ by targeting brain regions vulnerable to AD (Wirth et al., 2013b; Villeneuve et al., 2014). Neurodegenerative abnormalities in cortical thickness and glucose metabolism in AD-typical regions have, for instance, been associated with white matter lesions in cognitively normal older adults (Wirth et al., 2013b). While white matter lesions do not seem to interact with $A \beta$ to potentiate neurodegeneration (Haight et al., 2013), they nevertheless appear to have an additive impact on brain integrity (Chui et al., 2012). Vascular risk factors, particularly low levels of HDL cholesterol, have in turn been found to interact with $A \beta$ to reduce cortical thickness in AD-typical regions such as the precuneus, the temporoparietal associative cortex, and the superior and middle frontal cortices (Villeneuve et al., 2014). This interaction suggests that the impact of $A \beta$ on cortical thickness in AD-typical regions is potentiated in the presence of vascular risk (and/or vice versa). While $\mathrm{A} \beta$ deposition (La Joie et al., 2012) and its impact on neurodegeneration (Chételat et al., 2010; Villeneuve et al., 2014) is predominant in frontal and posterior association areas in individuals with cognitive impairments, vascular pathologies preferentially affect the frontal and temporal lobes (Jagust, 2013; Thal et al., 2014; Villeneuve et al., 2014). Therefore, brain regions such as the frontal lobe or the temporoparietal cortex represent converging points between $A \beta$ and vascular pathologies. Even if evidence is missing for an interaction between tau and vascular factors in $\mathrm{AD}$-typical regions, such interaction cannot be excluded and temporal regions such as the hippocampus are known to be vulnerable to both tau and vascular pathologies (Braak and Braak, 1991; Debette et al., 2011). Therefore, some AD-typical regions might also represent points of convergence between tau and vascular pathologies.

Figure 1 is a schematic illustration of our main hypothesis and does not represent real data. In Figure 1A, we propose that both the $A \beta$ and tau pathways are needed for $\mathrm{AD}$ clinical expression while other pathways such as the vascular pathway (related to vascular risk factors and/or vascular brain injuries) are not. Figure 1B represents brain regions most affected (injured) by each pathology in cognitively impaired individuals (Braak and Braak, 1991; Whitwell et al., 2008; Debette et al., 2011; La Joie et al., 2012; Villeneuve et al., 2014). It is important to stress that these maps probably vary from one individual to another as the effects of a pathology on brain integrity likely depend on the length of time the pathology has been present, the amount of pathology, the location of the pathology (particularly true for vascular brain injuries which can be more focal than tau and $A \beta$ ) as well as other genetic and environmental factors that could influence vulnerability to each pathological process. Also, it is extremely difficult to isolate the degree of importance of a single pathology since different pathologies frequently occur together and probably interact, as argued in this opinion paper. The AD-typical regions, shown as white dots in Figure 1C, represent brain regions that are typically atrophied 
and/or hypometabolic in individuals with AD (Dickerson et al., 2009; Landau et al., 2009; Schroeter and Neumann, 2011; Wirth et al., 2013a). In this opinion paper, we further suggest that they represent the point of convergence of multiple pathologies, as well as brain regions where pathologies might have a synergistic effect.

This viewpoint does not explain how one pathology may potentiate the other or why some brain regions might be more vulnerable to multiple pathologies (Seeley et al., 2009; Buckner and Krienen, 2013; Jagust, 2013). Rather, it stresses the importance of considering late-onset $\mathrm{AD}$ as a multi-factorial process and questions the notion that $A \beta$-negative individuals presenting atrophy or hypometabolism in $\mathrm{AD}$ typical regions are at low risk of $\mathrm{AD}$, especially if they are close to the threshold for $A \beta$-positivity. Indeed, if other pathologies interact with $A \beta$, low (or subthreshold) $A \beta$ levels could be sufficient to be harmful in the presence of these other pathologies. In conclusion, while $A \beta$ may be needed to develop the clinical symptoms associated with $\mathrm{AD}$, other factors might work together with $A \beta$ to promote brain injury in $\mathrm{AD}$-typical regions.

\section{GLOSSARY}

AD-typical regions $=$ limbic and heteromodal regions of the cerebral cortex typically found to be atrophied and/or hypometabolic in patients with dementia due to $\mathrm{AD}$.

\section{ACKNOWLEDGMENTS}

This work was supported by a Canadian Institutes of Health Research post-doctoral fellowship to SV, a Swiss National Science Foundation post-doctoral fellowship to MW, and by Philippe Chatrier Foundation and Thérèse et René Planiol Foundation post-doctoral fellowships to RLJ. We thank Jacob Vogel, Manja Lehmann, Natalie Marchant, John Breitner, Susan Landau, Gaël Chételat, and William Jagust for their comments on this manuscript.

\section{REFERENCES}

Aizenstein, H. J., Nebes, R. D., Saxton, J. A., Price, J. C., Mathis, C. A., Tsopelas, N. D., et al. (2008). Frequent amyloid deposition without significant cognitive impairment among the elderly. Arch. Neurol. 65, 1509-1517. doi:10.1001/archneur.65.11.1509

Braak, H., and Braak, E. (1991). Neuropathological stageing of Alzheimer-related changes. Acta Neuropathol. 82, 239-259. doi:10.1007/BF00308809
Buckner, R. L., and Krienen, F. M. (2013). The evolution of distributed association networks in the human brain. Trends Cogn. Sci. 17, 648-665. doi: 10.1016/j.tics.2013.09.017

Chételat, G. (2013). Alzheimer disease: a betaindependent processes-rethinking preclinical AD. Nat. Rev. Neurol. 9, 123-124. doi:10.1038/nrneurol. 2013.21

Chételat, G., Villemagne, V. L., Bourgeat, P., Pike, K. E., Jones, G., Ames, D., et al. (2010). Relationship between atrophy and beta-amyloid deposition in Alzheimer disease. Ann. Neurol. 67, 317-324. doi:10.1002/ana.21955

Chui, H. C., Zheng, L., Reed, B. R., Vinters, H. V., and Mack, W. J. (2012). Vascular risk factors and Alzheimer's disease: are these risk factors for plaques and tangles or for concomitant vascular pathology that increases the likelihood of dementia? An evidence-based review. Alzheimers Res. Ther. 3, 36. doi:10.1186/alzrt98

Crary, J. F., Trojanowski, J. Q., Schneider, J. A., Abisambra, J. F., Abner, E. L., Alafuzoff, I., et al. (2014). Primary age-related tauopathy (PART): a common pathology associated with human aging. Acta Neuropathol. 128, 755-766. doi:10.1007/s00401-0141349-0

Debette, S., Seshadri, S., Beiser, A., Au, R., Himali, J. J., Palumbo, C., et al. (2011). Midlife vascular risk factor exposure accelerates structural brain aging and cognitive decline. Neurology 77, 461-468. doi:10.1212/WNL.0b013e318227b227

Desikan, R. S., Thompson, W. K., Holland, D., Hess, C. P., Brewer, J. B., Zetterberg, H., et al. (2014). The role of clusterin in amyloid-beta-associated neurodegeneration. JAMA Neurol. 71, 180-187. doi:10.1001/jamaneurol.2013.4560

Dickerson, B. C., Bakkour, A., Salat, D. H., Feczko, E., Pacheco, J., Greve, D. N., et al. (2009). The cortical signature of Alzheimer's disease: regionally specific cortical thinning relates to symptom severity in very mild to mild AD dementia and is detectable in asymptomatic amyloid-positive individuals. Cereb. Cortex 19, 497-510. doi:10.1093/ cercor/bhn113

Fjell, A. M., McEvoy, L., Holland, D., Dale, A. M., Walhovd, K. B., and Alzheimer's Disease Neuroimaging Initiative. (2013). Brain changes in older adults at very low risk for Alzheimer's disease. J. Neurosci. 33, 8237-8242. doi:10.1523/JNEUROSCI.5506- 12. 2013

Fortea, J., Vilaplana, E., Alcolea, D., Carmona-Iragui, M., Sánchez-Saudinos, M. B., Sala, I., et al. (2014). Cerebrospinal fluid beta-amyloid and phosphotau biomarker interactions affecting brain structure in preclinical Alzheimer disease. Ann. Neurol. 76, 223-230. doi:10.1002/ana.24186

Haight, T. J., Landau, S. M., Carmichael, O., Schwarz, C., DeCarli, C., Jagust, W. J., et al. (2013). Dissociable effects of Alzheimer disease and white matter hyperintensities on brain metabolism. JAMA Neurol. 70, 1039-1045. doi:10.1001/jamaneurol. 2013.1878

Jagust, W. (2013). Vulnerable neural systems and the borderland of brain aging and neurodegeneration. Neuron 77, 219-234. doi:10.1016/j.neuron.2013. 01.002

Knopman, D. S., Jack, C. R. Jr, Wiste, H. J., Weigand, S. D., Vemuri, P., Lowe, V., et al. (2012). Short-term clinical outcomes for stages of NIA-AA preclinical
Alzheimer disease. Neurology 78, 1576-1582. doi: 10.1212/WNL.0b013e3182563bbe

La Joie, R., Perrotin, A., Barré, L., Hommet, C., Mézenge, F., Ibazizene, M., et al. (2012). Regionspecific hierarchy between atrophy, hypometabolism, and beta-amyloid (Abeta) load in Alzheimer's disease dementia. J. Neurosci. 32, 16265-16273. doi:10.1523/JNEUROSCI.2170-12.2012

Landau, S. M., Harvey, D., Madison, C. M., Koeppe, R. A., Reiman, E. M., Foster, N. L., et al. (2009). Associations between cognitive, functional, and FDG-PET measures of decline in $\mathrm{AD}$ and MCI Neurobiol. Aging 32, 1207-1228. doi:10.1016/j. neurobiolaging.2009.07.002

Mormino, E. C., Betensky, R. A., Hedden, T., Schultz, A. P., Amariglio, R. E., Rentz, D. M., et al. (2014) Synergistic effect of beta-amyloid and neurodegeneration on cognitive decline in clinically normal individuals. JAMA Neurol. 71, 1379-1385. doi:10. 1001/jamaneurol.2014.2031

Prins, N. D., and Scheltens, P. (2015). White matter hyperintensities, cognitive impairment and dementia: an update. Nat. Rev. Neurol. 11, 157-165. doi:10.1038/nrneurol.2015.10

Schroeter, M. L., and Neumann, J. (2011). Combined imaging markers dissociate Alzheimer's disease and frontotemporal lobar degeneration - an ALE metaanalysis. Front. Aging Neurosci. 3:10. doi:10.3389/ fnagi.2011.00010

Seeley, W. W., Crawford, R. K., Zhou, J., Miller, B. L., and Greicius, M. D. (2009). Neurodegenerative diseases target large-scale human brain networks. Neuron 62, 42-52. doi:10.1016/j.neuron. 2009.03.024

Small, S. A., and Duff, K. (2008). Linking Abeta and tau in late-onset Alzheimer's disease: a dual pathway hypothesis. Neuron 60, 534-542. doi:10.1016/ j.neuron.2008.11.007

Spillantini, M. G., and Goedert, M. (2013). Tau pathology and neurodegeneration. Lancet Neurol. 12, 609-622. doi:10.1016/S1474-4422(13)70090-5

Thal, D. R., Attems, J., and Ewers, M. (2014). Spreading of amyloid, tau, and microvascular pathology in Alzheimer's disease: findings from neuropathological and neuroimaging studies. J. Alzheimers Dis. 42(Suppl. 4), S421-S429. doi:10.3233/JAD- 141461

Villeneuve, S., Reed, B. R., Madison, C. M., Wirth, M., Marchant, N. L., Kriger, S., et al. (2014). Vascular risk and Abeta interact to reduce cortical thickness in AD vulnerable brain regions. Neurology 83, 40-47. doi:10.1212/WNL.0000000000000550

Whitwell, J. L., Josephs, K. A., Murray, M. E., Kantarci, K., Przybelski, S. A., Weigand, S. D., et al. (2008) MRI correlates of neurofibrillary tangle pathology at autopsy: a voxel-based morphometry study. $\mathrm{Neu}$ rology 7,743-749. doi:10.1212/01.wnl.0000324924. 91351.7d

Wirth, M., Oh, H., Mormino, E. C., Markley, C., Landau, S. M., and Jagust, W. J. (2013a). The effect of amyloid beta on cognitive decline is modulated by neural integrity in cognitively normal elderly. Alzheimers Dement. 9, 687-698. doi:10.1016/j.jalz. 2012.10.012

Wirth, M., Villeneuve, S., Haase, C. M., Madison, C. M. Oh, H., Landau, S. M., et al. (2013b). Associations between Alzheimer disease biomarkers, neurodegeneration, and cognition in cognitively normal older people. JAMA Neurol. 70, 1512-1519. doi:10. 1001/jamaneurol.2013.4013 
Conflict of Interest Statement: The authors declare that the research was conducted in the absence of any commercial or financial relationships that could be construed as a potential conflict of interest.

Received: 13 October 2014; accepted: 11 March 2015; published online: 25 March 2015.
Citation: Villeneuve S, Wirth $M$ and La Joie $R$ (2015) Are AD-typical regions the convergence point of multiple pathologies? Front. Aging Neurosci. 7:42. doi: 10.3389/fnagi.2015.00042

This article was submitted to the journal Frontiers in Aging Neuroscience.

Copyright (c) 2015 Villeneuve, Wirth and La Joie. This is an open-access article distributed under the terms of the Creative Commons Attribution License (CC $B Y)$. The use, distribution or reproduction in other forums is permitted, provided the original author(s) or licensor are credited and that the original publication in this journal is cited, in accordance with accepted academic practice. No use, distribution or reproduction is permitted which does not comply with these terms. 